\title{
Estimation of the power absorbed by the surface of optical components processed by an inductively coupled plasma torch
}

Renaud Jourdain, Marco Castelli, Mustapha Gourma, Nan Yu, Paul Shore,

Cranfield University, School of Aeronautic Transport and Manufacturing, Precision Engineering Institute, Cranfield, MK43 $O A L, U K$

Cranfield University, School of Energy, Environment and Agrifood, Cranfield University, Oil and Gas Engineering Centre, MK43 OAL, United Kingdom

r.jourdain@cranfield.ac.uk

\begin{abstract}
The focus of this work is the determination of the heat flux function -thermal footprint- of a plasma jet generated by an inductively coupled plasma (ICP) torch. The parameters of the heat flux function were determined through the correlation of modelling and experimental results. One surface of substrates was exposed to an impinging jet while the temperature changes of the unexposed surface was recorded, analysed and used to derive the parameters of the heat flux function. From a modelling viewpoint, a series of finite element analyses (FEA) were carried out to predict temperatures of substrate surfaces. From an experimental viewpoint, the plasma torch was powered by a $1 \mathrm{KW}$ radio frequency signal generator tuned at $39 \mathrm{MHz}$. The ICP torch equipped with a De-Laval nozzle impinged the surfaces of selected substrates at atmospheric pressure. Three sets of experiments - static, single pass and multi passes- were carried out to determine and validate the numerical description of the plasma jet. Also this work enabled to determine the maximum intensity of the heat flux distribution and the total power absorbed by substrate surfaces. Finally, the most advanced numerical model was used to assess the effect of a bi-directional raster scanning strategy that was used for the processing of large optical components.
\end{abstract}

Keywords: Impinging jet, local heat transfer, moving plate, nozzle plate

\section{Introduction}

Atmospheric plasma torches $[1,2,3]$ are commonly used in modern engineering and especially in surface treatment $[4,5]$. These plasma treatments are often based on dwell time methods and require a controlled exposure to obtain a uniformly treated surface [6-7]. One of these processes is called Plasma Figuring [8] and makes use of a bespoke inductively coupled plasma (ICP) torch [9]. The determination of the heat flux function of one type of these dedicated ICP torches is the motivation of this work.

The heat flux -thermal footprint- function investigated in this paper is provided by a modified ICP torch. The plasma torch is equipped with a convergent divergent nozzle that generates a turbulence free plasma jet that contains various species. Thus, species carried by the plasma jet travel at subsonic velocities in a well collimated manner until they impinge on the processed surface. This design was chosen to increase the efficiency -velocity and collimation- of the plasma jet. 
In the context of large optics fabrication, this ICP torch enables the local etching of silicon based materials [10]. Argon plasma is created and maintained through intense electro-magnetic fields induced by the RF signal applied to the coil -excitation region-. Then the central volume of the plasma receives the reactive species -fluorine compounds- that are ionised. The torch schematic is displayed in Figure 1.

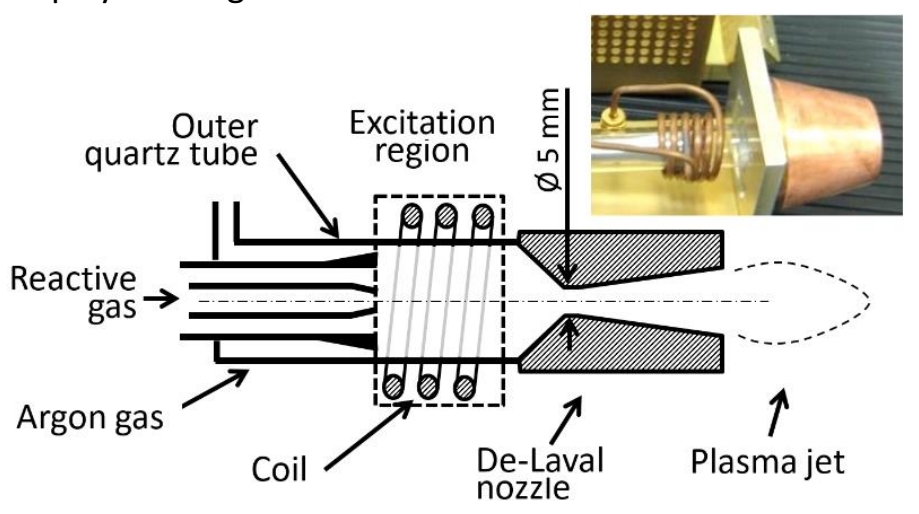

Figure 1. ICP torch equipped with a De-Laval nozzle

An extensive literature review was carried out to understand the existing numerical approach and current state of the art for both high temperature and isothermal unconfined axisymmetric jets.

The study of high temperature jets have motivated Mostaghimi [11], Dresvin and Amouroux [12] and Chen [13] amongst others. Mostaghimi focused on heat transfer in plasma spray coating processes. Dresvin described the general concept on convective heat transfer of jet and then applied it onto plasma jets. Chen's research results were about heat transfer and flow into a radio frequency plasma torch. However, the bespoke torch investigated in this paper is characterised by a design that none of the aforementioned research have addressed yet.

The flow structures of isothermal jets impinging flat surface motivated a huge number of studies in the 70 s and 80 s $[14,15,16]$. The understanding of their thermo-fluid-dynamic features was further highlighted by Carlomagno [17] and Viskanta [18] . Figure 2 illustrates both aerodynamic and thermodynamic characteristics of an isothermal jets.
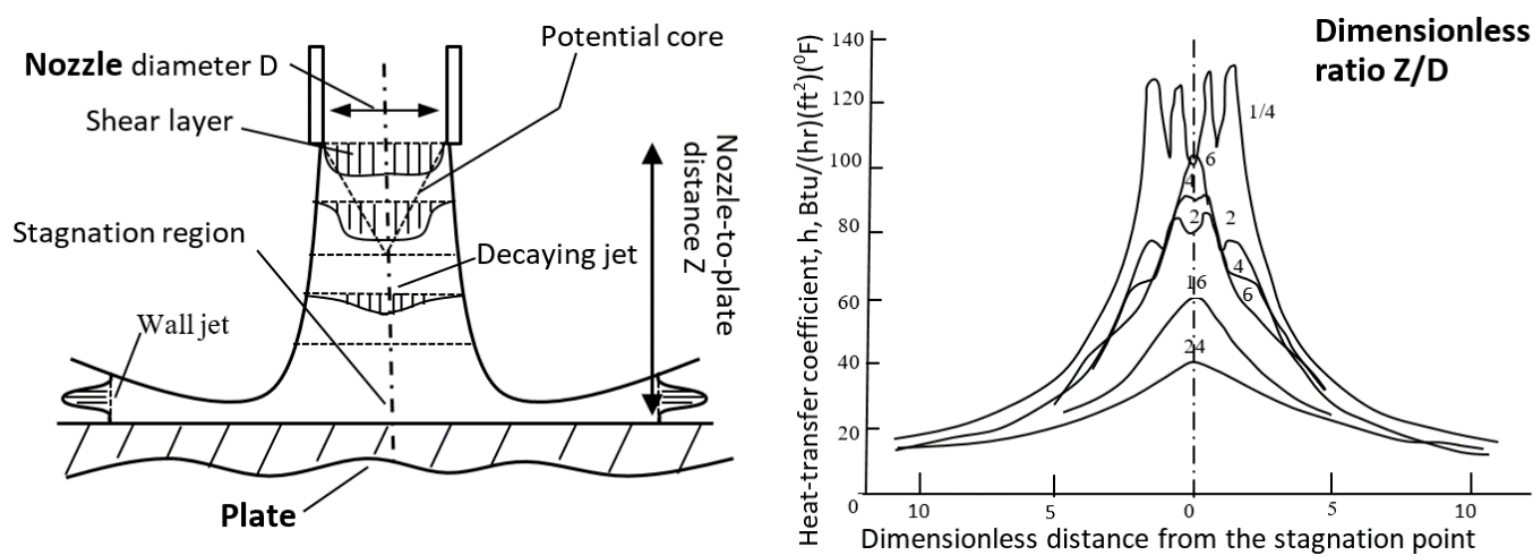

Figure 2. Flow regions of an impinging circular jet (left), radial profile of heat transfer coefficient between a plate and a impinging jet (Nozzle diameter $D=0.3634 \mathrm{~cm}, \mathrm{Re}_{\mathrm{D}}=28000$ ) (right) [19]

- Figure 2 (left) illustrates the aerodynamic characteristics of an impinging jet that passes through four different regions [19] some of which exist only for certain nozzle-to-plate distance $Z$ for a given diameter $D$. Indeed, when the nozzle-to-plate distance is reduced the jet fully developed region starts to disappear and then the whole decaying jet region vanishes. This disappearance happens for $Z / D<6$. If $Z / D \sim 2$, the high static pressure in the 
stagnation region has a significant influence on the flow development up to the nozzle exit. Therefore the usual quasi-conical shape of the core region disappears [17].

- Figure 2 (right) illustrates the radial profile of the heat transfer coefficient. The heat transfer coefficient profile is mainly influenced by the nozzle-to-plate distance over the diameter $Z / D$ ratio. For large ratio values $-Z / D>10$ - the radial profile has a bell shape distribution. For intermediate ratio values -i.e. $6<\mathrm{Z} / \mathrm{D}<10$ - a peak appears near the centre and the averaged heat transfer value increases to its maximum for about ratio value equal six. For small ratio value -i.e. $3<Z / D<6$ - a secondary annular peak is present. Extra small ratio value -i.e. $0.25<Z / D<2$ lead to relatively high heat transfer coefficients where impingement jet becomes wall jet [17].

The complex dimensionless number that represents the ratio of convective heat transfer over the conductive one is named the Nusselt number. Equation 1 enables to describe two important cases: the plate dimensions are close to or less than jet diameter, the plate dimensions are larger than jet diameter.

$N u=C \operatorname{Re}^{n}(Z / D)^{m}(L / D)^{k} \operatorname{Pr}$

where $C, n, m$ and $k$ are coefficients that were determined experimentally. A complete list of results can be sourced in Zuckerman and Lior work [20]. These coefficients depend largely on the experimental configurations. $Z$ is nozzle-to-plate distance. $D$ is the nozzle diameter. $L$ is the plate dimension. Re is the Reynolds number. Pr is the Prandl number.

$R e=\rho \vee D_{H} / \mu$

where $\rho$ is the density of the fluid, $v$ is the mean velocity of the fluid, $D_{H}$ is the hydraulic diameter of the nozzle, $\mu$ is the dynamic viscosity of the fluid.

$P r=C p v / k$

where $C_{p}$ is the heat capacity per unit volume of the fluid, $v$ its kinematic viscosity, and $k$ its thermal conductivity.

Dresvin and Amouroux [12] described analytically the heat transfer mechanism using a Newtonian formulation then they introduced the concept of enthalpy because the gas temperature in plasma jet is well above $3,000{ }^{\circ} \mathrm{C}$. From a numerical modelling viewpoint, high temperature jet research required either direct numerical simulations (DNS) or large eddy simulations (LES) [21]. These methods bring information that is not available using conventional CFD methods [22].

In a previous study carried out by the authors [9], the jet of the bespoke ICP torch nozzle was characterised in term of Reynolds number, temperature, velocity and pressure. Unlike in this paper, the plasma torch was operated using twenty percent more power and the nozzle-to-plate distance was $1.5 \mathrm{~mm}$ shorter. Whatever, two results obtained are worth to mention for the understanding of the unconventional modelling approach used in this paper. The Reynolds numbers ranged from 3,800 to 10,350 . The gas temperatures in the nozzle ranged from $6,500{ }^{\circ} \mathrm{C}$ by the inlet down to 1,500 ${ }^{\circ} \mathrm{C}$ by the outlet. Although some experimental parameters are different, it is certain that the jet is axisymmetric but is not isothermal. Also, this nozzle confines the jet through its long divergent section. These specifications are the reasons for which neither analytical nor numerical approaches mentioned in this introduction can be used.

In this work, the heat flux transferred by the bespoke ICP torch was managed by a motion controller that was used to implement raster scan toolpath algorithms [8]. The plasma torch was moved along the $x$ axis whereas the substrate is moved along the $y$ axis. Figure 3 (left) illustrates a snapshot of the torch and the workpiece $-420 \mathrm{~mm}$ side to side- in the processing chamber. Figure 3 (right) shows the bi-directional raster scan toolpath that was used for the validation of the numerical models (section 4). 



Figure $3.400 \mathrm{~mm}$ diameter optical mirror processed using the bespoke plasma torch

\section{Material and methods}

\section{1. Description of experiments}

The experimental approach chosen to determine the thermal footprint of the ICP torch was based on three sets of experiments. These experiments involved three optical materials (dielectrics) that did not affect the characteristics of the plasma jet unlike metallic material would do. The initial surface roughness value of all substrates was less than $2 \mathrm{~nm}$ Ra (average roughness). The chosen materials were silicon, low expansion glass and silicon carbide. These three materials have very different thermal properties [23] (Table 1). The substrates were instrumented for temperature measurements [24] (section 3.1 and 3.2).

The first set of experiments consisted of dwelling the plasma torch for duration of one to five seconds. The authors named this test "static exposure". The second set of experiments involved the displacement of the ICP torch at constant velocity along the $x$ axis direction. The authors named this test "single pass exposure". For these two sets of experiments, all three materials were used. The materials were fibre composite silicon carbide (MF type), single crystal silicon wafer (100 crystallographic orientation), and ULE (Ultra Low Expansion) glass. These are identified as substrate 01,02 and 03 respectively (Figure 4).

The third set of experiments was named "multi pass exposure". These experiments required only one material -ULE glass- (Figure 4 bottom right substrates 03 and 04 ) and the implementation of a toolpath algorithm that enabled the torch to raster scan the large $200 \mathrm{~mm} \times 200 \mathrm{~mm}$ surface (Section 3.2).

\section{2. Description of substrates used for experiments}

The pictures of the four substrates, dimensions and material types are provided in Figure 4. 


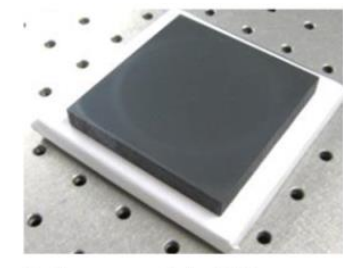

Substrate 01: $\mathrm{SiC}$ $75 \times 75 \times 10 \mathrm{~mm}$

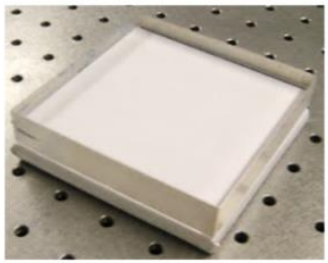

Substrate 03: ULE (1) $100 \times 100 \times 20 \mathrm{~mm}$

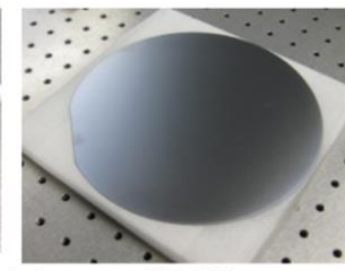

Substrate 02: Silicon

$\emptyset 200 \times 0.75 \mathrm{~mm}$

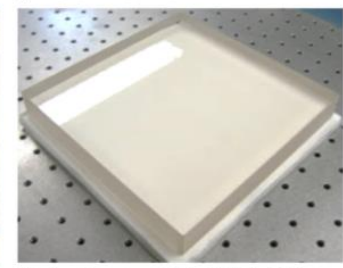

Substrate 04: ULE (2)

$200 \times 200 \times 25 \mathrm{~mm}$

Figure 4. Pictures of the four substrates, dimensions and material types

The thermal properties of the selected materials are provided in Table 1 and Figure 5. Values are displayed for the range of temperatures from $20^{\circ} \mathrm{C}$ up to $350^{\circ} \mathrm{C}$.

Table 1. Thermal material properties of substrates. The range of each parameter is given from low to high temperature $\left(20^{\circ} \mathrm{C}\right.$ up to $\left.350^{\circ} \mathrm{C}\right)$

\begin{tabular}{|c|l|c|c|c|c|}
\hline & Mubstrate & $\begin{array}{l}\text { Thermal } \\
\text { conductivity } K \\
\left(\mathrm{~W} \cdot \mathrm{m}^{-1} \cdot \mathrm{C}^{-1}\right)\end{array}$ & $\begin{array}{l}\text { Heat } \\
\text { capacitance } \mathrm{Cp} \\
\left(\mathrm{J} \cdot \mathrm{Kg}^{-1} \cdot \mathrm{C}^{-1}\right)\end{array}$ & $\begin{array}{l}\text { Density } \rho \\
\left(\mathrm{Kg} / \mathrm{m}^{3}\right)\end{array}$ & $\begin{array}{l}\text { Thermal } \\
\text { diffusivity } a \\
\left(10^{-6} \mathrm{~m}^{2} / \mathrm{s}\right)\end{array}$ \\
\hline \hline 01 & SiC $^{\mathrm{a}}$ & $121-50$ & $700-1440$ & 2650 & $65-13$ \\
\hline 02 & Silicon $^{\mathrm{b}}$ & $150-57$ & $705-870$ & 2330 & $91-28$ \\
\hline $03 \& 04$ & ULE $^{\mathrm{c}}$ & 1.31 & 767 & 2200 & 0.77 \\
\hline
\end{tabular}

a Fibre composite silicon carbide (SiC) type MF [25]

b Single crystal silicon (100 orientation) $[26,27]$

c ULE Ultra Low Expansion glass [28]
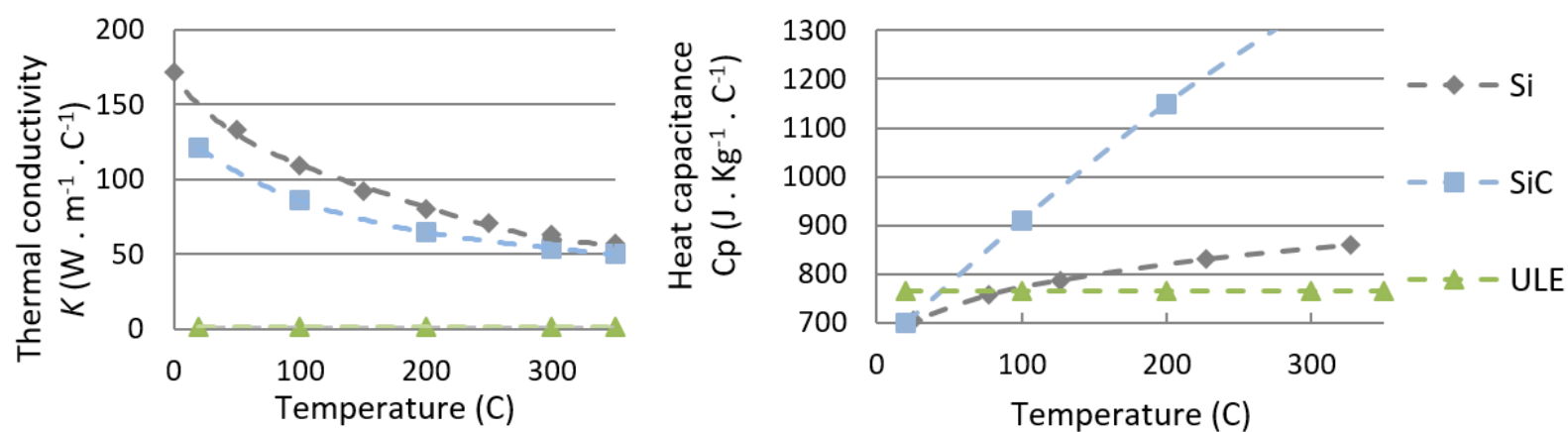

Figure 5. Thermal conductivity (left), and heat capacitance (right) of the three materials selected

\section{3. Plasma torch}

The plasma jet was generated at atmospheric pressure using an ICP torch (Figure 1). The plasma of the torch was altered by the use of a De-Laval nozzle [9]. This feature modified the characteristic of the jet and consequently its thermal footprint. The torch operating parameters are displayed in Table 2. These parameters were consistently used to obtain the experimental results. Also the fixed match RF network was frequently tuned to maintain the high energy coupling between the RF signal generator and the plasma jet. Finally, the quartz tubes were regularly replaced to ensure the symmetry of the plasma jet and reduce losses of the electromagnetic fields. 
Table 2. Parameter of the plasma torch

\begin{tabular}{ll}
\hline Signal frequency & $39.6 \mathrm{MHz}$ \\
Forwarded power & $1,000 \mathrm{~W}$ \\
Argon main flow & $20 \mathrm{I} / \mathrm{min}$ \\
Argon assist flow & $0.92 \mathrm{I} / \mathrm{min}$ \\
Reactive gas & $\mathrm{SF}_{6}$ \\
Reactive gas flow & $0.08 \mathrm{I} / \mathrm{min}$ \\
Nozzle(end)-to-plate & $7.5 \mathrm{~mm}$ \\
Nozzle(throat)-to-plate & $36.1 \mathrm{~mm}$ \\
\hline
\end{tabular}

\section{Experimental apparatus}

3. 1. Experimental configurations for static and single pass exposure experiments The instrumentations of substrates 01,02 , and 03 were carried out by mean of $\mathrm{K}$ type thermocouples (TC) [29]. Surfaces not exposed to the plasma jet were instrumented (Figure 6). Temperature changes were measured during both the exposure and cooling down duration of the experiments. All TCs were embedded in thermal paste and held in position by means of 70 um thick Kapton tape and 100 um thick aluminium tape. Figure 6 and Figure 7 display the schematic of the instrumentation and the position of the thermal footprint.

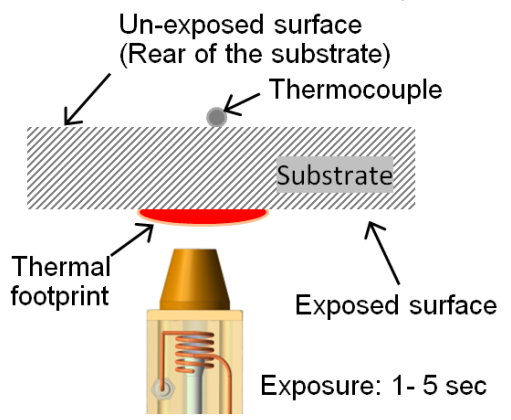

Figure 6. Schematic of the static exposure experiments

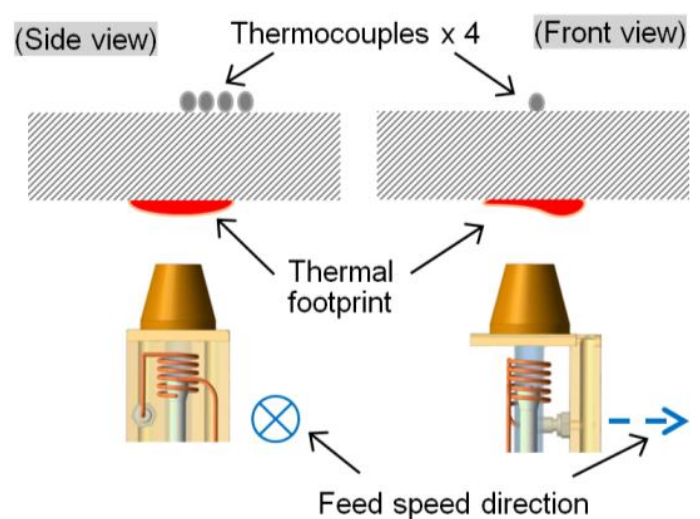

Figure 7. Schematic of the single exposure experiments

\section{2. Experimental configuration for multi pass exposure}

The large ULE test piece (substrate 04 Figure 4) was raster scanned using the ICP torch. The impinging plasma jet of the torch transferred the heat through a series of consecutive passes that were all parallel to each other's (Figure 8). The raster scan starting position was located $55 \mathrm{~mm}$ away from the edge parallel to the first travel path and $62.5 \mathrm{~mm}$ away from the edge orthogonal to the travel direction ( $x$ axis). The torch travelled at constant velocity set to $4 \mathrm{~m} \cdot \mathrm{min}^{-1}$. The total distance was $325 \mathrm{~mm}$ (left to right). Then the plasma jet moved backward by crossing the surface in the 
opposite direction (right to left). The pattern was repeated for completing the width of the raster scanning area. The raster scanned width was $90 \mathrm{~mm}$. The pitch of the raster scanning was either 1 $\mathrm{mm}$ or $2 \mathrm{~mm}$.



Figure 8. Tool path schematic for multi pass exposure experiments

For all experiments, the plasma torch was positioned below the processed surfaces. The plasma jet was pointing upwards and the distance between the top of the torch nozzle and the substrate surface was checked prior each run. The nozzle-to-plate distance was $7.5 \mathrm{~mm}$. The $25 \mathrm{~mm}$ thick ULE substrate was mounted in a bespoken holder to ensure that only the lower surface was exposed to the plasma jet.

The instrumentation of the large ULE substrate was carried out using K type TCs. The positions of these TCs were chosen for measuring the temperature changes onto both exposed and un-exposed surfaces of the substrate (Figure 9). Temperature records lasted 25 minutes while the processing substrate exposure to the plasma torch- lasted 3.5 and 7 minutes for the $2 \mathrm{~mm}$ and $1 \mathrm{~mm}$ raster scan pitch respectively. For accuracy and repeatability reasons, all three areas of interest were instrumented with two TCs. All TCs were embedded in thermal paste and held in position by means of two layers of Kapton tape and two layers of aluminium tape.

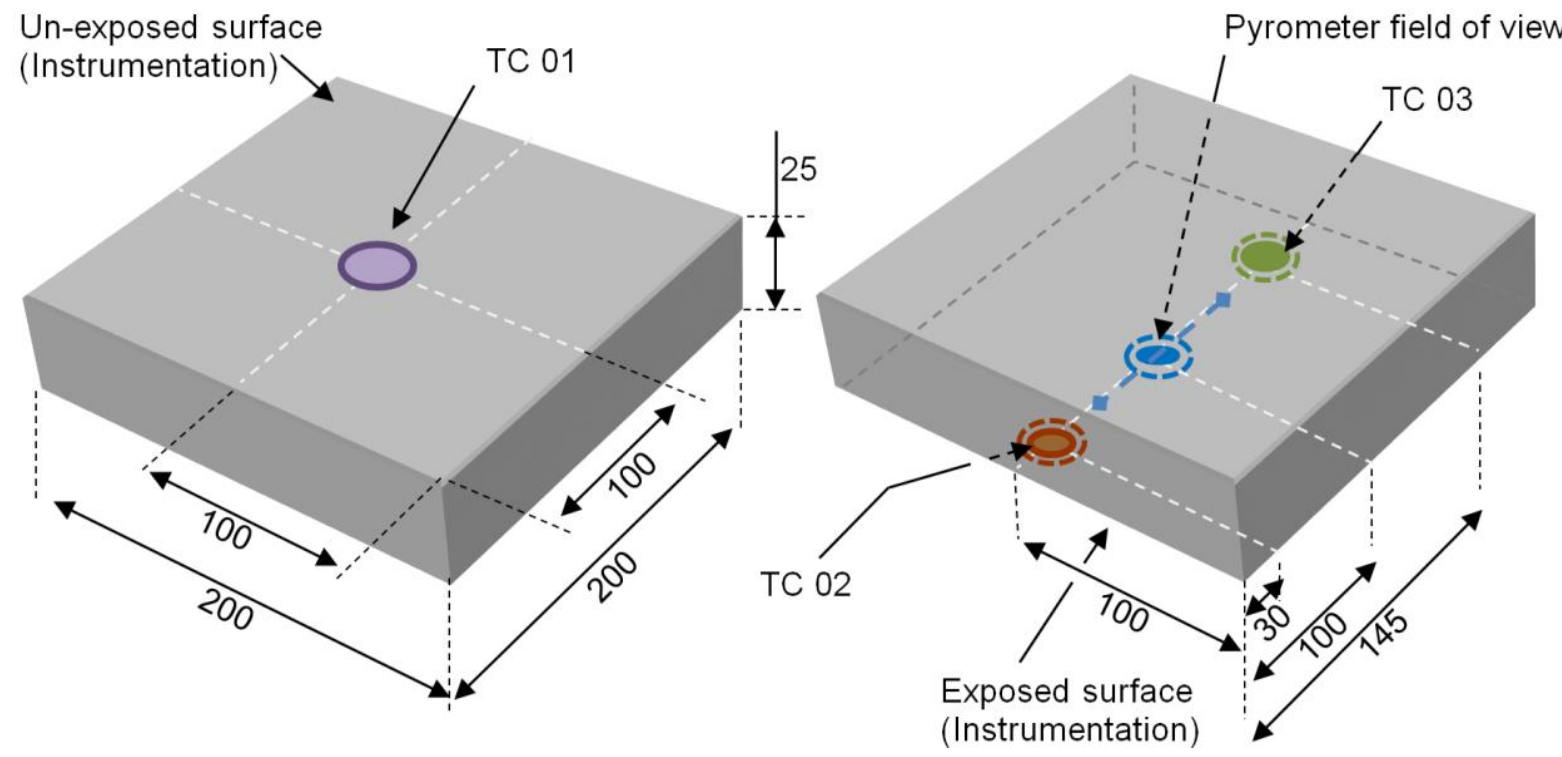


Figure 9. Position of the TC 01 on the un-exposed surface (left), positions of the TCs 02,03 and field of view area of the pyrometer on the exposed surface (right)

Local surface temperatures of the area exposed to the plasma jet were logged using a pyrometer $8 \mu \mathrm{m}$ to $14 \mu \mathrm{m}$ wavelength spectral bandwidth- [30] because the position of this area changes continuously. The pyrometer sensor head was maintained at $15 \mathrm{~mm}$ away from the surface and was mounted on the plasma torch carrier at a distance of $112.5 \mathrm{~mm}$. This setup enabled to prevent the pyrometer measurements to be affected by the plasma or the room illumination. Also the emissivity of the pyrometer was set to 0.95 after comparing its response with TCs positioned near the pyrometer field of view area. The acquisitions were carried out systematically mid distance to the substrate surface for every odd passes during each $90 \mathrm{~mm}$ raster scan. A total of 46 and 23 temperature measurements were carried out for $1 \mathrm{~mm}$ and $2 \mathrm{~mm}$ pitch respectively.

\section{Numerical modelling}

\section{4. $1 . \quad$ Modelling approach}

Two sets of novel numerical models were created using the commercial FEA software Ansys version 14. These models solved conduction problems using Fourier's law and Newton's law of cooling. Indeed, the radiation was ignored because the gas temperature is just above the thousand degree Celsius mark. The first and the second sets were created to predict temperature increase for static exposure and single pass exposure respectively. Models were two dimensional, transient, and nonlinear. The elements were quadratic and the mesh was mapped. A bespoke numerical thermal footprint was created to describe the plasma jet thermal footprint.

4. 2. Boundary conditions

a) Geometry and material properties of the substrates

The substrate geometries and dimensions are provided in Figure 4. The material properties are provided in Table 1and Figure 5.

b) Heat flux footprint of the plasma jet

This numerical heat flux footprint was characterised by four parameters: gas temperature, convection coefficient distribution type, convection coefficient distribution FWHM, convection coefficient maximum intensity.

- The gas temperature of the impinging jet was known to be in the range between $1,000{ }^{\circ} \mathrm{C}$ and $1,500{ }^{\circ} \mathrm{C}$ [9]. Therefore, the gas temperature value was set to $1,250{ }^{\circ} \mathrm{C}$. This choice is supported by Gordon results [31] who highlighted that turbulent jets reduce strongly the stagnation region dimension and favour constant temperature of the gas at the wall.

- The heat flux distribution type was set to be Gaussian. This choice was based on the results published by Jiang [32]. Also, according to Goldstein [33], the Bell shape distribution appears when Z/D ratio is equal or greater than six. The Z/D ratio of the bespoke jet was 7.22. Also, this choice was supported by analysing the single pass experimental results.

- The full width at half maximum (FWHM) of the Gaussian distribution of convection coefficient was set to $16 \mathrm{~mm}$. This value was determined in an empirical manner through the single pass dynamic experiments carried out on the silicon substrate (section 5.2 and Figure 17).

$F W H M=2 \sqrt{2 \operatorname{Ln}(2)} \sigma$

where $\sigma$ is the standard deviation. 
- The maximum intensity of the convection coefficient distribution was determined empirically. This value was inferred from the static exposure experiments carried out on the SiC and ULE substrates (section 5.1 and Figure 15).

The authors highlight that the numerical heat flux distribution was applied on surface cells through a bespoke sub routine that automatically accommodated for mesh cell changes and enabled to apply rigorously the same heat flux distribution parameters into all models.

c) Environment of the substrates

An investigation was carried out to determine the convection coefficient value for cooling down the substrates when set into the processing chamber. To do so, a $200 \mathrm{~mm} \times 200 \mathrm{~mm}$ ULE substrate was heated up to $100{ }^{\circ} \mathrm{C}$ and then it was left to cool down surrounded by ambient air. The temperature of the ambient air was measured and maintained at $20^{\circ} \mathrm{C}$ during this experiment. Experimental results matched the Newton's law of cooling [34]. The cooling down convection coefficient for a horizontally positioned substrate was determined to be $10 \mathrm{~W} \cdot \mathrm{m}^{-2} . \mathrm{C}^{-1}$. This result is in agreement with the range of values mentioned in publication [35].

\section{3. Mesh independency}

The dimension of the mesh inner cell was calculated using the quarter of the Fourier number. When this method was not possible then the spatial discretisation of the models were tested using regular meshing refinement procedure. In all cases, the mesh independence was ensured.

\section{4. Numerical model created for static exposure}

For the static exposure experiments, the authors created two dimensional axisymmetric models. The authors calculated a pseudo radius value for substrates 01 and 03 because the real substrates have a near square shape. This method provided an equivalent thermal mass for these substrates.

Figure 10 left displays the convection coefficient and temperature distributions used for the convection based model. The heat flux has a pseudo Gaussian profile because the calculation took into account the difference of temperatures between gas and surface.

The local heat flux changed from cell to cell for each step of transient calculation. The main benefit of this numerical approach is to decrease the intensity of the thermal footprint during the calculation when the substrate temperature has increased. This modelling approach describes closely the interaction between the substrate and the jet whatever the respective temperatures.



Figure 10. Thermal footprint for static exposure: convection and temperature profiles (left), Heat flux profile (right)

A second approach was the use of the regular heat flux distribution (Figure 10 right). This modelling approach did not require setting a gas temperature. Such approach was less rigorous but enabled to issue a number that explicitly determined the total amount of power transferred through the substrate surface. In the following equation 
$Q_{\text {Total }}=\frac{A}{2 \pi \sigma^{2}} \int_{0}^{\infty} e^{\frac{-(r-\mu)^{2}}{\left(2 \sigma^{2}\right)}} \int_{0}^{2 \pi} r d \theta d r$

where $A$ is the heat flux maximum intensity. $2 \pi \sigma^{2}$ is the normalisation coefficient. $\sigma$ is the standard deviation. $\mu$ is the offset distance from the edge of the substrate. $\theta$ is the rotational angle. $r$ is the radial distance.

\section{5. Numerical model created for single pass exposure}

The single pass exposure model was created using a plan two dimensional approach. As in the previous model, the boundary condition of each cell of the exposed surface was applied using the bespoke convection based Gaussian heat transfer distribution. Figure 11 shows the straight trajectory of torch for a single pass exposure. It was assumed that all cross sections received the same amount of heat. Both numerical and experimental results were extracted from the lower central point of the cross section located onto the surface unexposed by the torch jet. Thus the edge effect was neglected.

The relative intensity of the heat transfer function was calculated using the feed speed parameter. Indeed, this calculation is implicit and requires to change the maximum intensity of the heat flux distribution for each iteration. This intensity was dependant of the virtual position of the heat flux distribution along the scan direction.

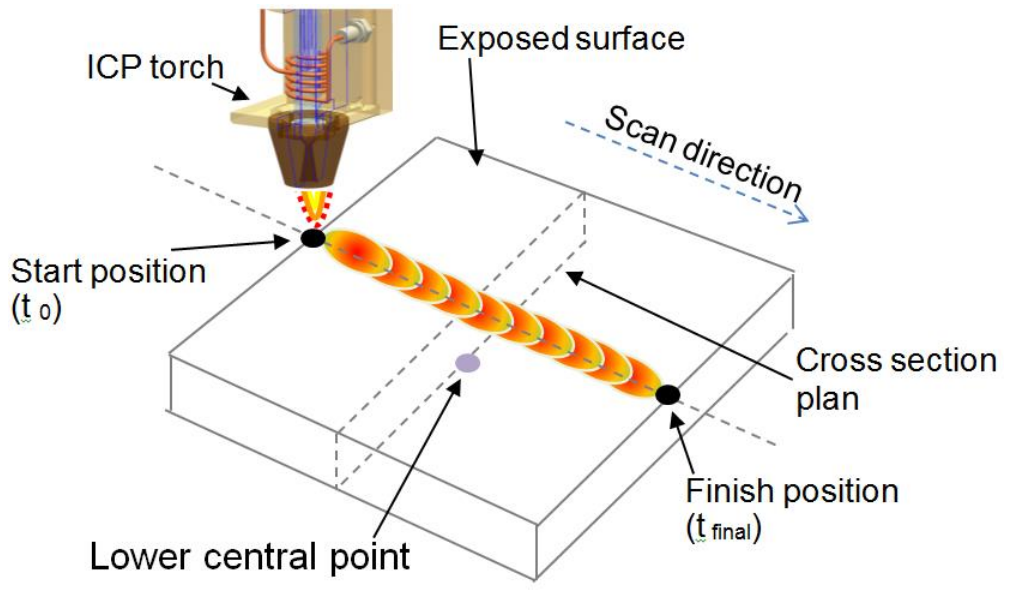

Figure 11. Single pass exposure

\section{6. Numerical model created for multi pass exposure}

Multi pass exposure model required the implementation of an additional routine for shifting the thermal footprint in the $Y$ direction. Indeed, the thermal footprint was re-applied after a calculated duration related to raster scan parameters such as feed speed, stroke length and pitch distances. This duration was based on the time between two consecutive passes (Figure 8). This bespoke thermal footprint was applied at set times when the torch is physically crossing the middle cross section of the substrate.

\section{Results}

There are three set of result sections in this paper. All sections present a correlation between experimental and numerical results. The first section is about static exposure tests. The second section is about dynamic exposure tests carried out through single pass exposures. The third section is about the dynamic heat transfer exposure tests carried out through multi pass exposures. 
5. 1. Substrates exposed to static heat source (static exposures)

The first set of results focuses on the temperature increases while the plasma jet dwelled for 5 seconds onto the substrate surfaces. The local temperature increase is plotted in the figures below for substrates: 01, 02, and 03.



Figure 12. Surface temperature versus time. Measurement carried out at the rear of substrate 01 (SiC)

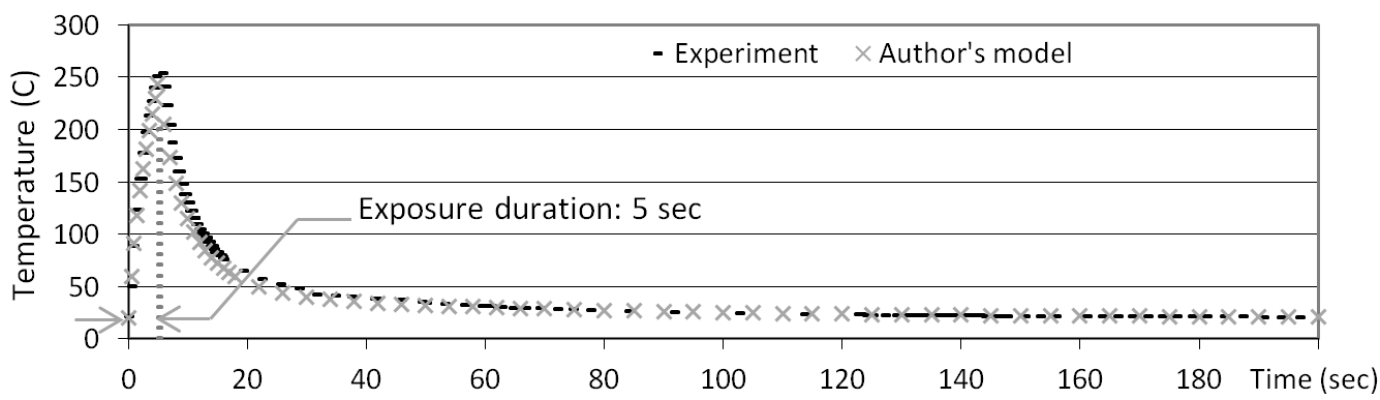

Figure 13. Surface temperature versus time. Measurement carried out at the rear of substrate 02 (Silicon)



Figure 14. Surface temperature versus time. Measurement carried out at the rear of substrate 03 (ULE)

The second set of results highlighted the maximum increase of temperature for exposure durations that were set from one up to five seconds (Figure 15).
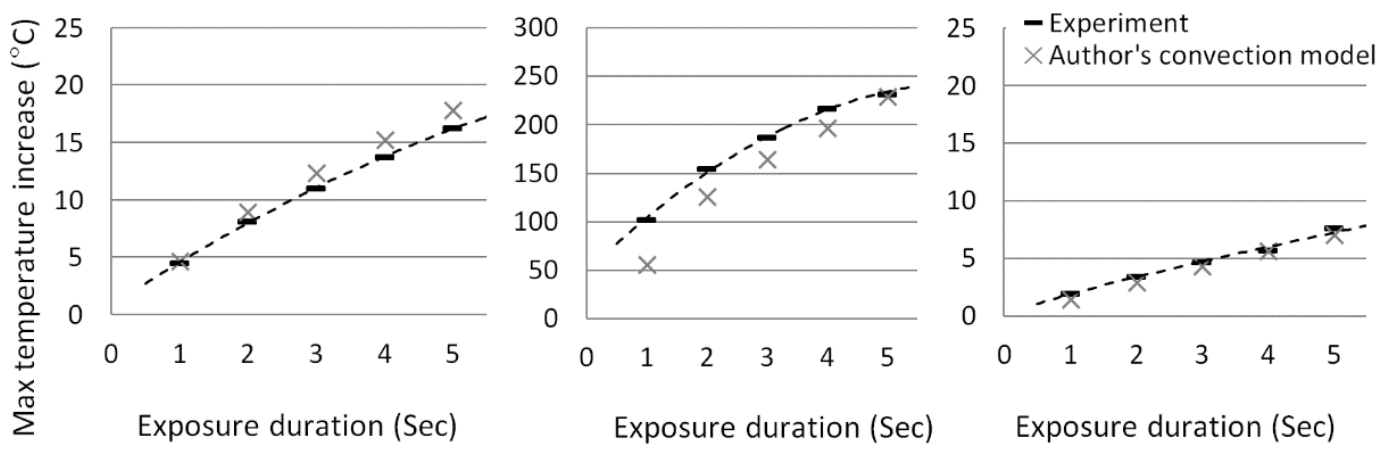

Figure 15. Maximum temperature increase versus exposure duration: SiC (left), silicon (middle): ULE substrate 03 (right)

The convection based model did slightly either over or under estimate the static experimental results. The mean errors for the maximum temperature increases were $0.40{ }^{\circ} \mathrm{C}, 1.14{ }^{\circ} \mathrm{C}$ and $23.8{ }^{\circ} \mathrm{C}$ for tests carried out on ULE, SiC, and silicon substrate respectively. The author's believe that 
measurements carried out on the silicon substrate using TCs were affected by the infrared emission spectrum of the plasma jet. Indeed, this material is characterised by a small absorption coefficient in this spectrum range [36].

This work highlighted the benefit of acquiring surface temperatures for duration much longer that exposure duration when dealing with glass materials because the thermal diffusivity values were small. The maximum temperature increase of the unexposed surface was often recorded minutes after the exposures. Figure 16 (upper half) shows temperature variations on $3 / 4$ isometric views after $5 \mathrm{sec}$ exposure duration. Figure 16 (lower half) shows the duration required for reaching a pseudo thermal equilibrium both through and across the substrate volumes.

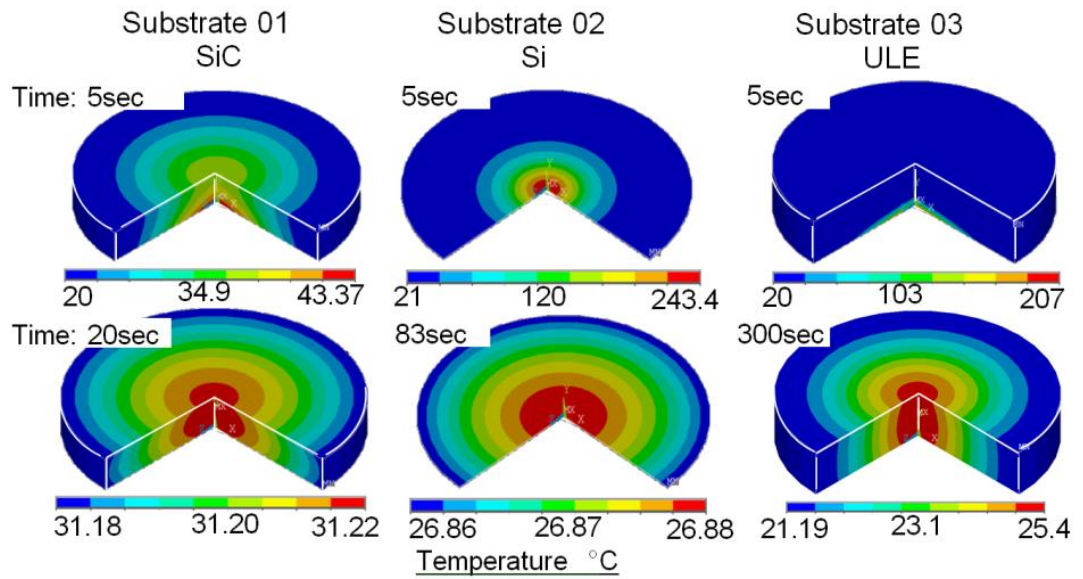

Figure 16. Temperature variations in substrates, $3 / 4$ isometric view: SiC (left), silicon (middle): ULE substrate 03 (right)

\section{2. Substrates exposed to a thermal footprint in motion (single pass exposures)}

This section focuses on the temperature increases while the plasma torch was moved at constant feed speed. Feed speed and TCs directions were orthogonal. Two sets of results are shown (Figure 17 and Figure 18).

The first result is about the increases of surface temperature that were both measured and modelled on the un-exposed surface of a silicon wafer. A set of four thermocouples -positioned 5 $\mathrm{mm}$ apart one to the others- enabled to measure the spatial temperatures along a line perpendicular to the torch travel direction (Figure 7 left). The temperature records were made when the torch was positioned precisely below the TC set. The repeat of this test led to the temperature profile shown Figure 17.

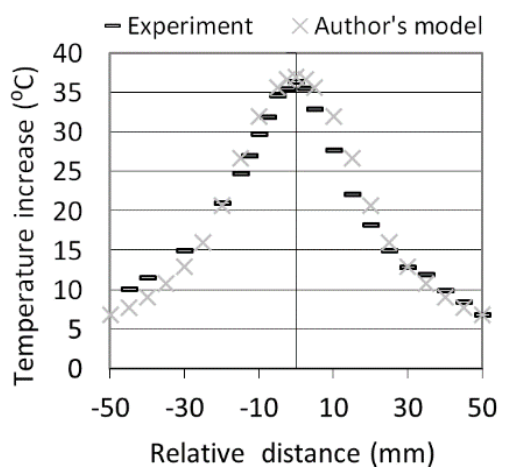

Figure 17. Temperature increases during a dynamic test on Si substrate (feed speed: $6 \mathrm{~m} \cdot \mathrm{min}^{-1}$ )

The second set of results focuses on the maximum temperature increases for feed speeds that ranged from $2 \mathrm{~m} \cdot \mathrm{min}^{-1}$ up to $8 \mathrm{~m} \cdot \mathrm{min}^{-1}$ (Figure 7 right). Figure 18 shows results for both substrate 02 
(silicon) and substrate 03 (ULE). Correlation between experimental and numerical results is within a few degrees Celsius.
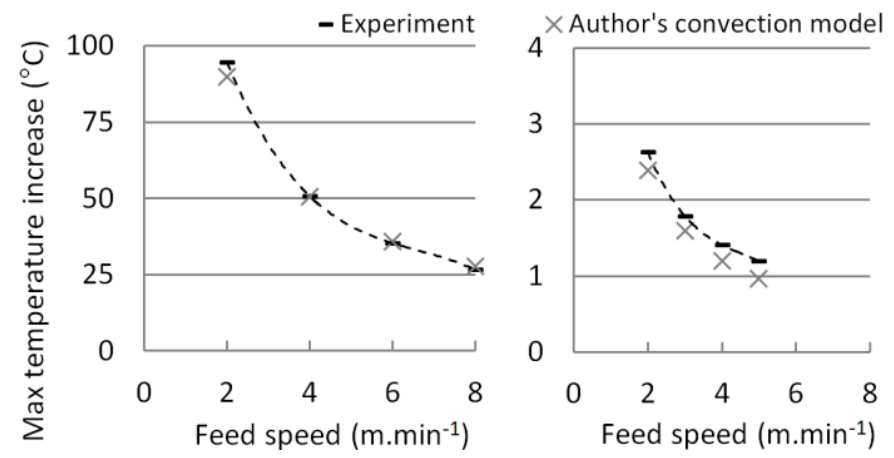

Figure 18. Maximum temperature increases versus feed speed: substrate 02 (silicon) (left), substrate 03 (ULE) (right)

\section{3. Raster scanning experiments (multi pass exposure)}

A further validation of the FEA model was carried out through two experiments that required the ICP torch to raster scan bi-directionally the surface of the substrate 04 (ULE). This experiment was significantly different compared to the one described previously (single pass exposures) because the thermal footprint is repeatedly applied onto the surface (Figure 8) and the surface is four time larger. Also, the temperature measurements were carried out at three different secured positions. Figure 19 show the results.
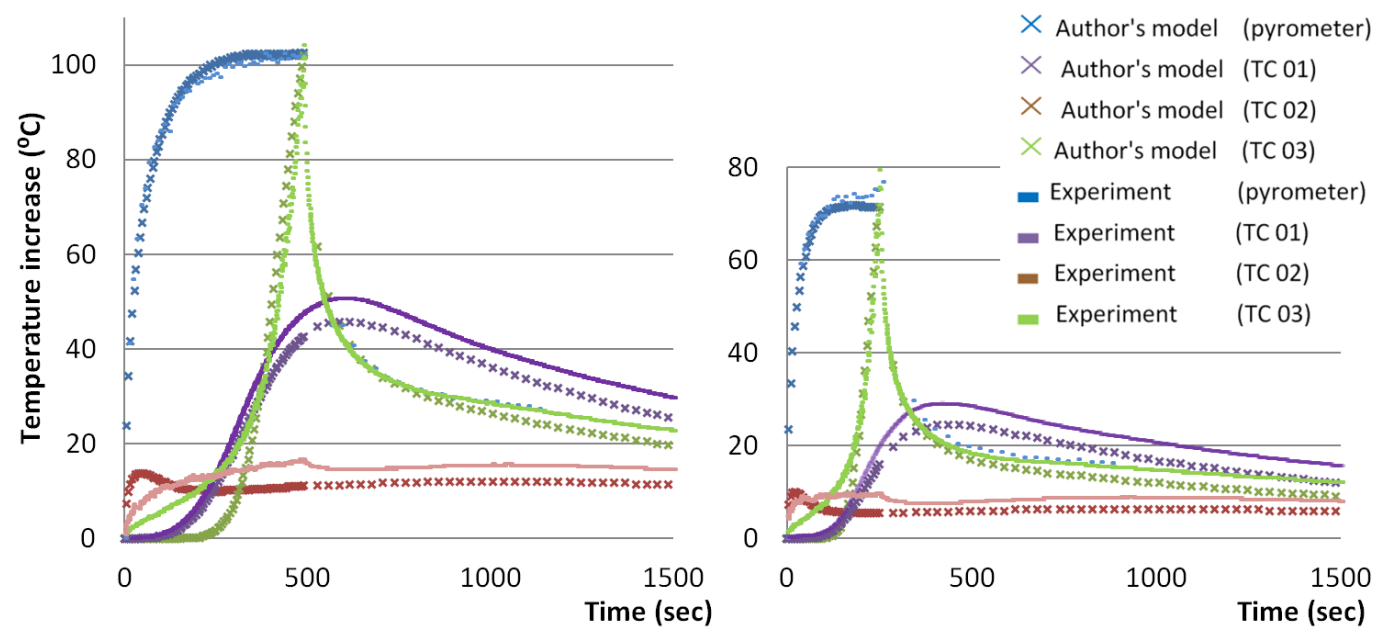

Figure 19. Logged and computed temperature versus time: $1 \mathrm{~mm}$ pitch (left), $2 \mathrm{~mm}$ pitch (right). Feed speed: $4 \mathrm{~m} . \mathrm{min}^{-1}$

Line graphs highlight very large temperature changes onto the different surface areas of the substrates. Results show that the exposed surface experienced a large temperature increase. Also the temperature increase of the area directly exposed by the plasma jet reached a platen value (blue crosses). A discrepancy between experimental and numerical results is noticeable. The authors consider that the heat from the torch affected the entire processing chamber and consequently decreased the cooling rate. 


\section{Discussions}

6.1. The combined experimental-modelling approach

This work demonstrated the benefit of carrying out different types of simple experiments and creating dedicated numerical models for the investigation of temperature changes caused by a plasma torch. Static and dynamic experiments contributed to quantitative and qualitative analysis respectively.

6.2. Determination of the heat flux parameters

All parameters of the heat flux distribution were determined through the novel approach presented in this paper. The results obtained using the thick low thermal conductivity substrate (ULE) and the thick high thermal conductivity substrate (SiC) contributed to determine the total amount of power absorbed. The results obtained using the thin high thermal conductivity substrate (silicon) contributed to determine the heat flux distribution profile type. On the other hand, the heat flux based model was used to derive the maximum intensity of the heat flux. The value was $13.7 \mathrm{~kW} . \mathrm{cm}^{-}$ 2

6.3. Need for non-linear numerical scheme

The implementation of a nonlinear modelling scheme is believed to be necessary even if most results showed temperature increases well below $100{ }^{\circ} \mathrm{C}$. The reason is that these results are shown for the surfaces un-exposed to the thermal footprint. The maximum temperatures increase, onto the surface exposed to the plasma jet, often reached $250{ }^{\circ} \mathrm{C}$ which fully justified the use of non-linear material properties.

\subsection{Nozzle efficiency}

From a fluid dynamic viewpoint, there is a positive assessment on the jet performance. The main reason is the ratio between nozzle-to-plate and jet diameter. Indeed, Z/D is equal to 7.2. This value is likely to favour both the existence of turbulence and a maximum stagnation point as reported by Gardon, and Akfirat [31]. This ratio is beneficial for the processing when the torch is used to remove material but this point of discussion is outside the scope of this paper.

From a thermal viewpoint, there is also a positive assessment on the jet performance. Indeed, the ratio $Z / D$ value affects greatly the amount of heat transferred into the substrate. Using equation 5 , it was found that $220 \mathrm{~W}$ from the original $1,000 \mathrm{~W}$ where transferred into the substrates. This value is very low. However it is known that regular ICP torches are roughly $50 \%$ efficient [37, 38]. Furthermore, this torch is equipped with a large water cooled De-Laval nozzle that absorbs more than ten percent of the supply power [39].

\subsection{Error analysis of the technique}

A potential source of errors that affected the experimental recordings were dominated by the perturbations due to the electromagnetic field of the RF plasma torch. However, the authors have developed their own expertise for shielding sensors, electrical leads and recording instruments using metal foils.

A numerical error was due to the set infinite dimension normal to the cross sections in the plan 2D numerical models used to predict single and multi pass exposure experiments. Indeed, ULE 03, ULE 04 and SiC substrates were $100 \mathrm{~mm}, 200 \mathrm{~mm}$ and $75 \mathrm{~mm}$ in length along the exposure direction. Then, the authors express a conservative view on this error. 
Other potential errors were due to the type of boundary conditions chosen for heat flux of the jet. In static conditions, the two approaches used to numerically describe the thermal footprint led to two sets of results. The use of heat flux based thermal footprint led to overestimate most experimental results (Figure 20). Indeed, when the substrate temperature is close to the gas temperature then the amount of heat transferred has to be reduced such as in the real physics. The mean errors for the maximum temperature increases of this model are $0.19{ }^{\circ} \mathrm{C}, 2.35{ }^{\circ} \mathrm{C}$ and $5.87{ }^{\circ} \mathrm{C}$ for tests carried out on ULE, SiC, and silicon substrates respectively.
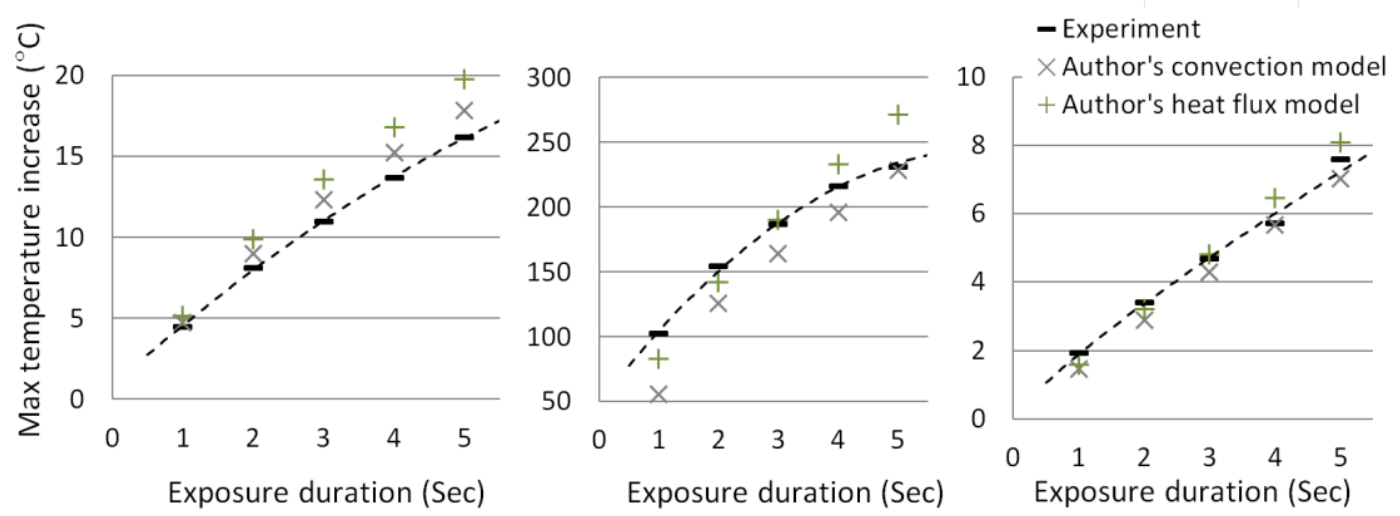

Figure 20. Maximum temperature increases versus exposure duration: SiC (left), silicon (middle): ULE substrate 03 (right)

Dynamic single pass experimental conditions required a different approach thus a plan two dimensional model was used. The surface temperature distribution predictions were within than half degree Celsius deviation from the experimental results.

6.6. Processing recommendations in the context of optical fabrication

The author's modelling approach enabled to predict the surface temperature increases occurred by a bi-directional raster scan toolpath. These temperature increases were predicted onto both exposed and un-exposed surfaces. These results highlighted that large substrates do experience significant surface temperature variations during and after processing. Results obtained are characteristic of a raster scan and un-forced natural cooling down of glass substrates. The correlation between the numerical model results and the recorded measurements is within a few degrees Celsius. Indeed, it is beneficial to create diagnostic tools for the identification of areas where thermally induced stresses exist because they can lead to failure of the processed component.

\section{Conclusion}

This work enabled the determination of the heat flux -thermal footprint- of a plasma jet generated by a bespoke ICP torch through a novel method.

The total power absorbed by the substrates was $220 \mathrm{~W}$ for an ICP torch equipped with De-Laval nozzle that is powered by $1 \mathrm{~kW}$ RF signal and positioned at a distance of $7.5 \mathrm{~mm}$ from an optical surface. The results showed that temperatures could be spatially and transiently predicted for both static and dynamic experimental conditions.

Then the effects of a bi-directional raster scan toolpath carried out on a large glass substrate were highlighted. Results revealed that local temperatures of the processed surface increase progressively before reaching a platen value. These results suggest that engineers should choose a specific toolpath strategy depending on application requirements. Indeed, uniform surface temperature will enable higher quality of the surface treatment. 


\section{Acknowledgments}

This research work was funded by the Cranfield Innovative Manufacturing Research Centre (20072012) of the Engineering and Physical Sciences Research Council (EPSRC) UK (GR/585337; EP/F031416/1). This publication was funded by the Centre for Innovative Manufacturing in Ultra Precision of the EPSRC UK (EP/I033491/1). 


\section{Biographies}

Dr Renaud Jourdain is an Academic Fellow in the Precision Engineering Institute of the School of Aeronautic Transport and Manufacturing (SATM) at Cranfield University. Since 2008, he has been instrumental in the development of a new fast figure correction method - based on the dwell time technique - for metre scale optical components. Dr Jourdain and his team have embraced the challenge of bringing this unique plasma based figuring process called the Plasma Figuring to a near industrial scale. Currently, Dr Jourdain is developing further his research activities in the field of energy beams used to structure and correct free form optical surfaces.

Dr Marco Castelli is a Development Engineer at Loxham Precision Ltd., a spin-out company of the Precision Engineering Institute at Cranfield University. During his PhD at Cranfield, he successfully conducted research focused on the development of dwell-time based tool-path codes for the Reactive Atom Plasma process. Currently, Dr Castelli is involved in various industrial projects, including automation and vision systems, as well as large optics fabrication, where he continues to work on algorithms and software development for manufacturing applications.

Pr Paul Shore, FREng whose background experience spreads across industry and academia, has coordinated the UK's $1^{\text {st }}$ DTI funded Link Nanotechnology project entitled "Ultra Precision Machining Research" in 1989. At $f 1.7$ million it was the largest project under this DTI programme. Prof Shore has also been responsible for Precision Production Engineering for the SKF Group working in the Netherlands and Sweden. Since 2002, he has worked at Cranfield establishing its Precision Engineering Institute as a world-leading research facility. In 2009, he was elected as a Fellow to the Royal Academy of Engineering; in the same year he was elected as the incoming President of the European Society for Precision Engineering and Nanotechnology (Euspen). 


\section{References:}

[1] M. Morsli, P. Proulx and D. Gravelle, Chemical non-equilibrium modelling of an argon-oxygen supersonic ICP, Plasma Sources Sci. and Technology 20 (2011).

[2] U. Kogelschatz, Atmospheric-pressure plasma technology, Plasma Phys. Controlled Fusion 46 (2004) B63-B75.

[3] M. I. Boulos, The inductively coupled R.F. (radio frequency) plasma, Pure and Appl. Chemistry 57 (9) (1985) 1321-1352.

[4] O. V. Penkov, M. Khadem, W.-S. Lim, D.-E. Kim, A review of recent applications of atmospheric pressure plasma jets for materials processing, J. Coating Technology and Res. 12 (2) (2015) 225-235.

[5] H. C. Chen, E. Pfender, and J. Heberlein, Improvement of plasma spraying efficiency and coating quality, Plasma Chemistry and Plasma Processing 17 (1) (1997) 93-105.

[6] P. Fauchais, Understanding plasma spraying, J. of Phys.D: Appl. Phys.37 (2004) R86-R108

[7] S.E. Selezneva, M.I. Boulos, Supersonic induction plasma jet modelling, Nucl. Instruments and Methods in Phys. Res. Section B: Beam Interaction with Materials and Atoms 180 (1-4) (2001) 306311.

[8] M. Castelli, R. Jourdain, P. Morantz, P. Shore, Fast figuring of large optics by reactive atom plasma, SPIE, Astronomical Telescopes + Instrumentation, vol. 8450 , art. nb. $34-134-8,1^{\text {st }}-6^{\text {th }}$ July, 2012.

[9] Y. Nan, R. Jourdain, M. Gourma, P. Shore, "Analysis of De-Laval nozzle designs employed for plasma surface figuring of Surfaces", The International J. of Advanced Manufacturing Technology, (2016) 1-11.

[10] R. Jourdain, M. Castelli, P. Shore, P. Sommer, D. Proscia, Reactive atom plasma (RAP) figuring machine for meter class optical surfaces, Production Eng. Res. and Development 7 (6) (2013) 665673.

[11] J. Mostaghimi, S. Chandra, Heat transfer in plasma spray coating processes, in A. Fridman, Y. Cho (Eds.), Transport Phenomena in Plasma, Advances in Heat Transfer, vol. 40, 2007, pp. 143-204.

[12] S. V. Dresvin, J. Amouroux, Heat and mass transfer in plasma jet, in A. Fridman, Y. Cho (Eds.), Transport Phenomena In Plasma, Advances in Heat Transfer, vol. 40, 2007, pp. 451-522.

[13] X. Chen, Heat transfer and flow in a radio frequency plasma torch-a new modelling approach, International J. of Heat and Mass Transf. 33 (5) (1990) 815-826.

[14] J. W. Gauntner, J. N. B. Livingood, P. Hrycak, Survey of literature on flow characteristic of a single turbulent jet impinging on a flat plate, NASA technical note, Lewis Research Center, February 1970.

[15] J. N. B. Livingood, P. Hrycak, Impingement heat transfer from turbulent air jets to flat plates - a literature survey, NASA technical note, Lewis Research Center, May 1973.

[16] P. Hrycak, Heat transfer from impinging jets, a literature review, New Jersey Institute of Technology, June 1981.

[17] G. M. Carlomagno, A. Ianiro, Thermo-fluid-dynamics of submerged jets impinging at short nozzle-to-plate distance: a review, Exp. Therm. and Fluid Sci. 58 (2014) 15-35.

[18] R. Viskanta, Heat transfer to impinging isothermal gas and flame jets, Exp. Therm. and Fluid Sci. 6 (1993) 111-134.

[19] R. Gardon, J. Cobonpue, Heat transfer between a flat plate and jets of air impinging on it, Int. Developments in Heat Transf., ASME (1963) 454-460.

[20] N. Zuckerman, N. Lior, Jet impingement heat transfer: physical, correlations, and numerical modelling, in G. A. Greene (Eds), Advances in Heat Transfer, vol. 39, 2006, pp. 565-632.

[21] A. Dewan, R. Dutta, B. Srinivasan, Recent trends in computation of turbulent jet impingement heat transfer, Heat Transfer Eng. 33 (2012) 447-460.

[22] X. Jiang, H. Zhao, K. H. Luo, Direct computation of perturbed impinging hot jets, Computers \& Fluids 36 (2007) 259-272. 
[23] R. Gardon, A Review of radiant heat transfer in glass, J. of the American Ceramic Society 44 (7) (1961) 305-312.

[24] H. J. Hodge, Temperature measurement in engineering, in H. C. Wolfe (Ed), Temperature. Its Measurement and Control in Sciences and Industry, vol. 3, Reinhold publishing corporation, New York, 1955 pp. 287-325.

[25] http://www.cesic.de/PDF/Datasheet_Cesic_MF_engl_06_2011.pdf (visited on 12/07/2016)

[26] C. J. Glassbrenner and G. A. Slack, Thermal conductivity of silicon and germanium from 3 to the melting point, Phys. Rev. 134 (4A) (1964) A1058-A1069.

[27] A. Okhotin, A. Pushkarskii, V. Gorbachev, Thermophysical properties of semiconductors, Moscow, Atomizdat (1972).

[28] T. W. Hobbs, M. Edwards, R. VanBrocklin, Current fabrication techniques for ULE and fused silica lightweight mirrors, SPIE vol. 5179 Bellingham, WA, (2003).

[29] T. J. Quinn, Temperature, monographs in physical measurement, Chap. 6, Academic Press, 1983.

[30] P. Saunder, Radiation Thermometry: Fundamental and Applications In The Petrochemical Industry, SPIE press book, 2007.

[31] R. Gardon, J.C. Akfirat, The role of turbulence in determining the heat transfer characteristics of impinging jets, International J. of Heat Mass Transf. 8 (1965) 1261-1272.

[32] X. Jiang, H. Zhao, K. H. Luo, Direct computation of perturbed impinging hot jet, Comp. and Fluids 36 (2007) 259-272.

[33] R. J. Goldstein, A. I. Behbahani, K. Heppelmann, Streamwise distribution of the recovery factor and the local heat transfer coefficient to an impinging circular jet, Int. J. Heat Mass Transfer 29 (1986) 1227-1235.

[34] M. I. Davidzon, Newton's law of cooling and its interpretation, Int. J. of Heat and Mass Transf. 55 (2012) 5379-5402.

[35] R. E. Bolz, CRC Handbook of Tables for Applied Engineering Science, CRC Press, 1973.

[36] G. G. Macfarlane, T. P. McLean, J. E.Quarrington, and V. Roberts, Exciton and phonon effects in the absorption spectra of germanium and silicon, J. Phys. Chem. Solids 8, (1959) 388-392.

[37] T. B. Reed, Induction-coupled plasma torch. J. of Applied Physics 32(5) (1961) pp.821-824.

[38] R.C. Miller and R.J. Ayen, Temperature profiles and energy balances for an inductively coupled plasma torch. Journal of Applied Physics 40(13) (1969) 5260-5273.

[39] N. Yu, R. Jourdain, M. Gourma, P. Shore, Investigation of power dissipation in a collimated energy beam, 6th Inter. Conf. of Asian Society for Precision Engineering and Nanotechnology (ASPEN), China, Harbin, 15th - 20th August, 2015 
2016-08-06

\section{Estimation of the power absorbed by the surface of optical components \\ processed by an inductively coupled plasma torch}

Jourdain, Renaud

Elsevier

Jourdain R, Castelli M, Yu N, Gourma M, Shore P. Estimation of the power absorbed by the surface of optical components processed by an inductively coupled plasma torch, Applied pÿThermal Engineering, Volume 108, 5 September 2016, Pages 13721382.

http://dx.doi.org/10.1016/j.applthermaleng.2016.08.024

Downloaded from Cranfield Library Services E-Repository 